\title{
Note on the Preparation of Sodium Amalgam in the Form of Pellets
}

\author{
H. S. Isbell, H. L. Frush, and N. B. Holt
}

(August 26, 1959)

A procedure is described for the preparation of sodium amalgam in the form of pellets.

In connection with the development of methods for the synthesis of radioactive carbohydrates, ${ }^{1}$ a procedure and apparatus were previously described $[1,2,3]^{2}$ for the reduction of semimicro quantities of aldonic lactones to sugars. The method employs sodium amalgam together with a slightly soluble, acid salt (sodium binoxalate) as buffer. ${ }^{3}$ The amalgam is used in the form of pellets made by dropping the molten amalgam into a "shot tower" of oil. Because other workers have had difficulty in making these pellets, the procedure is now given in detail.

The amalgam is prepared in a $500-\mathrm{ml}$, roundbottomed, stainless-steel flask having a single neck, with a 24/40 standard-taper joint (outer), ${ }^{4}$ and a thermometer inlet. The joint is fitted with a stainless-steel stopper, which has an inlet tube bent at right angles to the stopper and covered with asbestos for convenience in handling. The flask is held in a sturdy, asbestos-covered clamp, which serves as a handle for the vessel during the heating step. An alundum (Soxhlet extraction) thimble $45 \mathrm{~mm}$ in diam, in the bottom of which six 1.5-mm holes have been drilled, is held in a second asbestos-covered clamp. The oil bath is a thick-walled heat-resistant glass jar, 6 in. in diam and 18 in. high, containing paraffin oil to within 3 in. of the top (see fig. 1).

To prepare the amalgam, a weighed amount of mercury is placed in the flask, into which a continuous stream of dry nitrogen is passed by means of the inlet tube of the stopper. The required amount of sodium ${ }^{5}$ is weighed under paraffin oil, and then cut into pieces just small enough to be readily slipped through the neck of the flask. Each piece is rinsed

\footnotetext{
1 A project sponsored by the Division of Research of the Atomic Energy Commission.

2 Figures in brackets indicate the literature references at the end of this paper,

3 The reduction procedure has also been modified for use with macro quantities of lactones [4].

4 Scientific Glass Company, Bloomfield, N.J.

$\checkmark$ For the reduction of aldonic lactones under the conditions employed in this
} laboratory a 5 -percent amalgam has been found to be satisfactory. in a hydrocarbon solvent, such as heptane or toluene, quickly blotted dry, and dropped through the neck of the flask into the mercury; the stopper is immediately replaced. The sodium reacts quickly with the mercury and may be added fairly rapidly because of the atomosphere of nitrogen. After the addition of the sodium is completed, the flask is heated with a

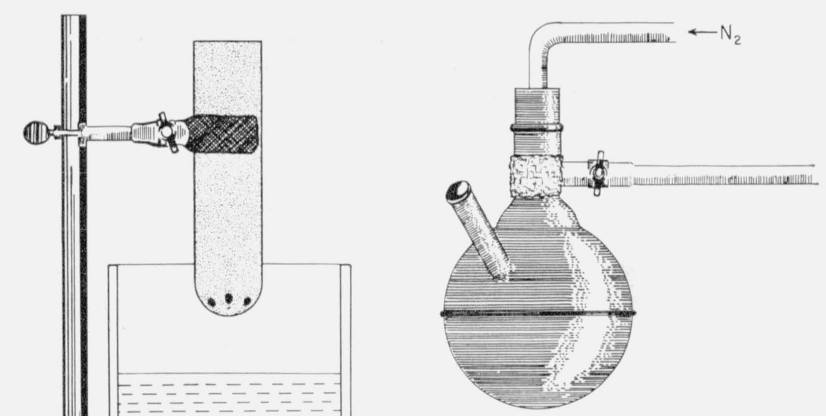

FIgURe 1. Apparatus used in the preparation of sodium amalgam pellets. 
Meeker burner until the amalgam is entirely molten. (The presence of remaining solid particles in the amalgam may be detected by the sound of their impact on the walls of the flask when it is given a gentle, swirling motion.) While the amalgam is being prepared, the alundum thimble is heated over another Meeker burner by a second operator. The hot thimble is then so clamped that its bottom is 1 to 2 in. above the surface of the oil, and the molten amalgam is poured into the thimble from the thermometer inlet of the flask. The amalgam flows through the holes in the bottom of the thimble, drops through the oil, and collects at the bottom of the oil bath as small, rather flat pellets.

Optimal conditions for the production of smooth pellets must be determined by trial. If the flask and thimble have not been sufficiently heated, the amalgam may solidify in the thimble. If the holes in the thimble are too large, the product may be somewhat "thready." However, once the optimal conditions have been established, the procedure may be repeated without difficulty.

The entire operation must be performed in an efficient hood. The amalgam is stored under paraffin oil in a wide-mouthed, screw-capped bottle (see fig. 2). Pellets are removed as needed, weighed under oil, and rinsed with an inert, volatile solvent immediately before use.

\section{References}

[1] H. S. Isbell, J. V. Karabinos, H. L. Frush, N. B. Holt, A. Schwebel, and T. T. Galkowski, J. Research NBS 48, 163 (1952) RP2301.

[2] H. S. Isbell, U.S. Patent No. 2,632,005, Mar. 17, 1953.

[3] H. L. Frush and H. S. Isbell, J. Research NBS 50, 133 (1953) RP2400.

[4] H. L. Frush and H. S. Isbell, J. Research NBS 54, 267 (1955) RP2588.

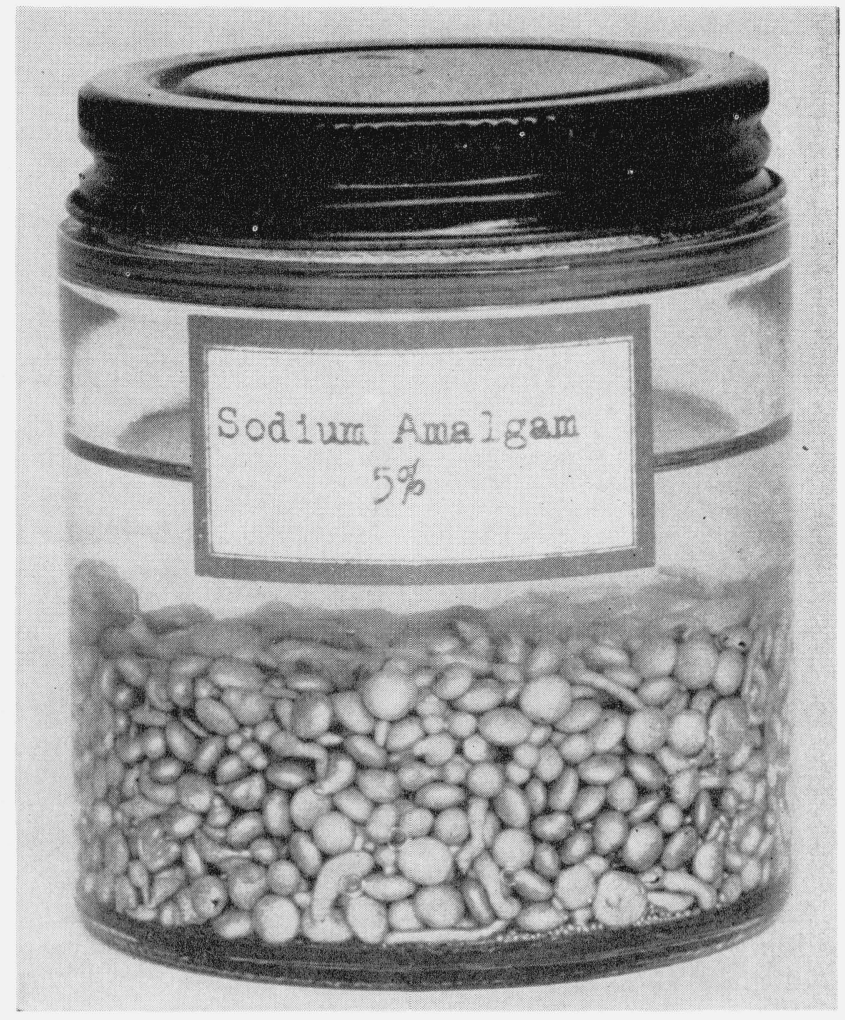

Figure 2. Sodium amalgam pellets.

Washington, D.C.

(Paper 64A1-35) 


\section{Selected Abstracts}

\begin{abstract}
Surface-wave resonance effect in a reactive cylindrical structure excited by an azial line source, A. L. Cullen, J. Research NBS 64D, No. 1, 13 (1960).
\end{abstract}

It is shown that a purely reactive cylinder excited by a neighboring line source can, under suitable conditions, give rise to a radiation pattern closely approximating the function $\cos n \theta$.

In a numerical example, a cylinder of three T.E.M. wavelengths circumference has a surface reactance chosen to emphasize the term $\cos 6 \theta$ in the Fourier series of the resultant radiation pattern. It is shown that only 1.1 percent of the total power delivered to the line source is radiated in unwanted modes.

It is also shown that the position of the line source does not affect this result to first order provided that $k(b-a)<<1$, where $b-a$ is the distance of the line source from the cylindrical surface.

Basic experimental studies of the magnetic field from electromagnetic sources immersed in a semi-infinite conducting medium, M. B. Kraichman, J. Research NBS 64D, No. 1, 21 (1960).

Using electromagnetic sources, consisting of various dipoles and loops immersed in a concentrated sodium chloride solution, measurements were made verifying the magnetic field propagation equations in air, derived previously by several authors. The receiver was farther away from the source than a wavelength in the conducting medium, but much closer than a wavelength in air

An expression is derived giving the value of the magnetic field in air due to a rectangular loop with a horizontal axis by assuming the loop to consist of two electric dipoles corresponding to the horizontal members. Experimental data verifying this expression are presented.

Also, using submerged electric dipoles, measurements were made of the magnetic field in air which show that the field is determined solely by the current in the horizontal radiating wires of the dipoles.

Determination of the amplitude-probability distribution of atmospheric radio noise from statistical moments, W. G. Crichlow, C. J. Roubique, A. D. Spaulding, and W. M. Beery, J. Research NBS 64D, No. 1, 49 (1960).

During the International Geophysical Year, the National Bureau of Standards established a network of atmospheric noise recording stations throughout the world. The ARN-2 noise recorder at these stations measures three statistical moments of the noise: average power, average voltage, and average logarithm of the voltage. An empirically derived graphical method of obtaining an amolitude-probability distribution from these three moments, and its development, is presented. Possible errors and their magnitudes are discussed.

Recorder survey: Recording surfaces and marking methods, G. Keinath, NBS Circ. 601, 41 (1959) 30 cents.

This Circular surveys the characteristics and comparative advantages of continuous traces, dotted traces, and printed characters, as produced by inking, incision, impression, indentation, deposition, heat, light, electric discharge, electron beam, magnetism, chemical action, or fluid streamlines. Descriptive and reference material is included on three physical components of the recording system-the reservoir of material or energy, the marking point or matrix positioned by the measuring element, and the chart surface which preserves the record.

Energy dissipation by fast electrons, L. V. Spencer, NBS Monograph 1, 70 (1959) 45 cents.

Tabulations are given of the energy dissipated by fast electrons at different distances from nomoenergetic electron sources, for plane perpendicular and point isotropic sources. A summary of the theoretical methods and data utilized, and a table of spatial moments are also included.

Calculated behavior of a fast neutron spectrometer based on the total absorption principle, J. E. Leiss, Tech. Note 10 (PB151369) \$1.00.

Performance calculations for a total absorption fast neutron spectrometer are presented. The spectrometer detecting element is a boron-10-loaded liquid scintillator. By making the scintillator a thin disk, only those neutrons whose first collision is a large-energy-loss hydrogen collision have appreciable chance of remaining in the spectrometer long enough to be captured. The expected energy resolution and efficiency of this type of spectrometer are determined, and are comparable to other types of fast neutron spectrometers.

Penetration of gamma rays from isotropic sources through aluminum and concrete, M. J. Berger and L. V. Spencer, Tech. Note 11 (PB151370) 50 cents.

Semianalytical expressions, with numerically specified parameters, are given which represent the gamma ray dose distribution in infinite aluminum or concrete media for sources that are monoenergetic (with energies between $10.22 \mathrm{Mev}$ and $0.0341 \mathrm{Mev})$, isotropic, and have the form of an infinite plane, point, disk, or spherical surface.

Precise time synchronization of widely separated clocks, A. H. Morgan, Tech. Note 22 (PB151381) \$1.50.

This paper describes known prescise methods of setting a group of widely separated clocks to precisely the same time and keeping them in close agreement indefinitely; most of the proposed methods are now available. An estimate of the accuracies of each method are given. Some discussion of high frequency radio propagation theory pertinent to two of the methods and a few sets of measurements of the propagation delay time of high frequency signals from WWV to WWVH are given. Several graphs and tables are included to simplify some of the calculations.

Design of single frequency filters, F. F. Fulton, Jr., Tech. Note 23 (PB151382) 50 cents.

Efficient procedures are shown for designing filters formed by a number of identical resonant circuits loosely coupled together, and which are required to accept one narrow band of frequencies and reject another narrow band somewhat removed in frequency without any special requirements on the shape of the attenuation curve in between these regions. The design is based on using a large number of sections.

Note on bivariate linear interpolation for analytic functions, W. Gautschi, Math. Tables and Other Aids to Computation 13, No. 66, 91 (1959).

A detailed study of the accuracy of bivariate linear interpolation is applied on real and imaginary parts of an analytic function with some suggestions for improving the accuracy. 
Absorption of radiation by a cylindrical sample of a strong absorber, P. H. Fang and I. A. Stegun, Letter to Editor, J. Chem. Phys. 31, 267 (1959).

The connection between the Bessel function and an integral which occurs in an absorption of radiation by a strong absorber is discussed. The effect of using this exact evaluation in the analysis of experimental data is indicated.

PILOT-A new multiple computer system, A. L. Leiner, W. A. Notz, J. L. Smith, and A. Weinberger, J. Assoc. Computing Mach. 6, No. 3, 313 (1959).

The Pilot data processor is a high-speed multiple computer system, more than 100 times faster than SEAC. It contains three interconnected computers for rapid processing of data, and also contains multiple input-output channels for rapid transfer of data into and out of the system. All of these units operate concurrently in a coordinated fashion. A summary description is given of the overall logical plan of the system including the principal characteristics of the first computer, the second computer, the third computer, the internal controls, and the external controls.

The system combines internal processing capabilities that are fast and versatile with external communication capabilities that are exceptionally flexible. This combination permits the internal power of the machine to be exploited readily by the outside world, either by other automatic devices or by human operators.

Analysis of vibrational relaxation data in shock wave experiments, K. E. Shuler, J. Chem. Phys. 30, No. 6, 1631 (1959).

In the analysis of the data on vibrational relaxation behind shock waves it is necessary to take account of the fact that more than the two lowest vibrational states may be appreciably populated. An analysis for systems with many quantum states is carried out for an ensemble of harmonic oscillators.

Branched-chain higher sugars. I. A 9-aldo4-C-formyl-nonose derivative, $R$. Schaffer and H. S. Isbell, J. Am. Chem. Soc. 81, 2178 (1959).

Two molecules of 5-aldo-1,2-O-isopropylidene-D-xylo-pentofuranose in alkaline solution combine to form a branchedchain decose derivative (I) by an aldol condensation. An explanation is offered for the occurrence of this condensation, which is not a typical carbohydrate reaction. A proof of the complete structure and configuration of $\mathrm{I}$ is presented and the compound is named 9-aldo-4-C-formyl-1,2:8,9-di- $O$-isopropylidine-L- $x y l o-\mathrm{L}-i d o$-nono-1, $4: 9,6$-difurano-4 (1), 7 - $\alpha$-pyranose.

The use of a vacuum microbalance in studies of electron tube materials, G. F. Rouse, Proc. $4^{\text {th }}$ Natl. Conf. on Tube Technigues, Sept. 10 to 12, 1958, Sponsored by the Advisory Group on Electron Tubes, p. 262 (New York University Press, New York, N.Y., 1959).

Several of the physical and chemical processes which occur in an electron tube involve changes of mass. For instance, sublimation from the cathode or breakdown of the coating decrease the cathode mass. Absorption or adsorption of gas by a tube part increases its mass. If change of mass can be measured with sufficient accuracy and the measured change can be correlated with controlled experimental conditions, a better understanding of the factors governing particular processes may be possible. This greater understanding may make it possible to control the processes so as to minimize any harmful effects which might arise from them.

A study based on this idea of measuring change of mass was initiated some time ago, a vacuum microbalance being the test device.
Effects of gamma radiation on collagen, J. Cassel, J. Am. Leather Chemists' Assoc. LIV, No. 8, $432(1959)$.

The effect of gamma radiation was determined on collagen exposed as a purified steerhide powder and as kangaroo tail tendon. Absorbed radiation ranged from 5 to 220 megarads. Apparent even at low radiation levels were the decrease in shrinkage temperature and increased water-solubility. The same decrease in shrinkage temperature was observed for tendons irradiated in the presence or absence (vacuum) of air. The shrinkage temperature was not influenced by the moisture content of the tendon during irradiation. Relatively little damage to amino acid structure was observed at absorption doses less than 20 megarads. The amino acid components most susceptible to alteration by radiation are methionine, phenylalanine, and threonine, while those least damaged were alanine, glycine, hydroxyproline, proline, and arginine. Radiation causes a nonhydrolytic type of peptide chain scission. High ultraviolet absorbance by the watersoluble fractions is due to formation of specific complex ultraviolet-absorbing groups rather than to light scattering.

Standards for neutron flux measurement and neutron dosimetry, R. S. Caswell, E. R. Mosburg, Jr., and J. Chin, 2d United Nations Intern. Conf. on the Peaceful Uses of Atomic Energy 21, Health and Safety: Dosimetry and Standards, P/752 USA, p. 92 (1959).

Known neutron fluxes for laboratory use may be obtained from sources in which neutrons are produced by a natural radioactivity. An absolutely calibrated radium-beryllium photoneutron source is used as a primary standard of source strength. Methods for intercomparisons and calibrations of neutron sources are given, together with the results of international source intercomparisons. A standard thermal neutron flux geometry, absolutely calibrated by $B^{10}(n, \alpha)$ reaction rate is described. Methods for separating neutron and gamma ray dose in mixed radiation fields by use of proportional counters with pulse-height discrimination are discussed.

A study of limb flares and associated events, C. Warwick and M. Wood, Astrophys. J. 129, No. 3, 801 (1959).

Measured heights of limb flares were used to derive a frequency distribution of flare height and to assess the importance of height in the production of flare associated events. The observed height distributions can be explained by a real distribution with maximum at zero, decreasing toward greater heights. The occurrence of SWF (shortwave fadeout) depends strongly on flare height. This dependence could be explained as an effect either of absorption of the ionizing radition in the sun's atmosphere or of a mechanism of production of ionizing radiation that depends critically on the height of the flare. Center-to-limb variation of SWF occurrence, while not conclusive, favors the former interpretation. The relation between optical flare characteristics and occurrence of associated bursts of radionoise is strongest for decimeter bursts and weaker for bursts at meter wavelengths.

Low even configurations in the first spectrum of ruthenium (Ru i), R. E. Trees, J. Opt. Soc. Am. 49, 838 (1959).

The theory of intermediate coupling with configuration interaction is used to calculate energy levels and $g$-values in the $4 d^{6} 5 s^{2}, 4 d^{7} 5 s$, and $4 d^{8}$ configurations of Ru I. By using 15 parameters, the positions of 57 observed levels are calculated with a mean deviation of $\pm 54 \mathrm{~cm}^{-1}$ between theory and experiment. There are unexplained differences greater than $500 \mathrm{~cm}^{-1}$ for two other levels, one of which is well-confirmed by experiment. Overall agreement between observed and calculated $g$-values is within \pm 0.006 . Linear relations are obtained between similarly defined electrostatic parameters in the three configurations. 
Magnetic study of the frozen products from the nitrogen microwave discharge, B. J. Fontana, J. Chem. Phys. 31, No. 1, 148 (1959).

Magnetic susceptibility measurements have been made during the deposition in a solid $\mathrm{N}_{2}$ matrix of nitrogen atoms produced in a microwave discharge. From the nitrogen atom concentration in the gas reaching the condensing surface and the magnetic measurements on the deposited solid, it is concluded that a concentration of about 0.2 to 0.5 mole percent $\mathrm{N}$ cannot be exceeded in the solid phase. Such concentrations are observed only for relatively brief intervals after which catastrophic recombination occurs. Stable, long-lived concentrations which account for the previously reported thermal and light emission effects which accompany warmup of such deposits are estimated to be less than 0.01 to 0.04 mole percent N. Susceptibility measurements are based on comparison with solid molecular oxygen and the calculation of $\mathrm{N}$ atom concentrations assumes that the susceptibility of dilute $\mathrm{N}$ atoms in an $\mathrm{N}_{2}$ matrix approximates that of the free gas.

* The thermal E.M.F. of several thermometric alloys, R. L. Powell and M. D. Bunch, Suppl. au Bull. inst. intern. froid (Delft, Holland),Comm. 1, 129 (1958).

Measurements of the thermal emf of Au-2.1 at. percent Co, Constantan and "normal" silver versus copper have been made from $4^{\circ} \mathrm{K}$ to $300^{\circ} \mathrm{K}$, with reference termperatures at the boiling points of liquid helium, liquid hydrogen, and liquid nitrogen. The uniformity of each type of thermo element was tested by simultaneous cablibration of several samples from the same melt.

Geometrical anistropy of magnetic materials in waveguides and cavities, L. A. Steinert, J. Appl. Phys. 30, 1109 (1959).

The perturbations caused by magnetized ferrite materials placed inside waveguides or resonant cavities depend upon the geometrical shape of the material as well as its intrinsic properties.

In the case of isotropic ferrite ellipsoids, a general formulation is given for the ratio of the radiofrequency magnetization $4 \pi \vec{m}$ of the material to the uniform radiofrequency field $\overrightarrow{h_{0}}$ external to the material. A few special examples are included.

Radioactivity standardization in the United States, W. B. Mann and H. H. Seliger, 2d United Nations Intern. Conf. on the Peaceful Uses of Atomic Energy 21, Health and Safety: Dosimetry and Standards, $P / 750$ USA, p. 90 (1959).

Recent developments are described in radioactivity standardization by compensated gas counting and liquid scintillation counting.

Magnetic interaction of $\mathbf{H}_{3}, \mathrm{~V}$. Griffing, J. L. Jackson, and B. J. Ransil, J. Chem. Phys. 30, No. 4, 1066 (1959).

The results of an LCAO-MO-SCF-CI calculation of the ${ }^{2} \Sigma_{u}$ ground state of linear $\mathrm{H}_{3}$ are presented and the wave functions obtained from this calculation are used to calculate the general features of the electron spin resonance spectrum. The calculation is carried through for the Fermi contact interaction, which is the principal interaction between the proton and electron spins. It is found that the off-diagonal elements of the hyperfine interaction matrix may not be neglected in that they split degenerate levels. The predicted spectrum, however, does not agree with the observed electron spin resonance spectrum of discharged hydrogen deposited at liquid helium temperatures. In addition the expected electron spin resonance spectrum of $\mathrm{H}_{2}{ }^{+}$has been calculated from the exact wave function.
The nature, cause and effect of the porosity in electrodeposits. IV. Influence of gas bubbles on the formation of pores, F. Ogburn and D. W. Ernst, Plating 46, 957 (1959).

One mechanism for the formation of a pore in electrodeposits involves the inclusion of a surface gas bubble. Whether the pore becomes continuous or bridged-over depends upon the relative rates of hydrogen discharge and nickel deposition. A cell is described which permits the observation of a simulated "cross section" of a nickel deposit while it is being formed. A series of photomicrographs obtained with this cell shows the enclosing of a gas bubble.

The nature, cause and effect of the porosity in electrodeposits. V. An evaluation of the sensitivity of the ferroxyl test, F. Ogburn, D. W. Ernst, and W. H. Roberts, Plating 46, 1052 (1959).

The importance of a good porosity test prompted the determination of the sensitivity of the ferroxyl test. Strausser's modification of the test was used to determine the porosity of the gold coatings on steel. A photographic process determined the porosity of goldfoil obtained by stripping the steel away with acid. A filar micrometer was used to measure the size of the pores present in the goldfoil. A comparison of the porosities of the goldfoil as determined by both methods gave the following results for pores between 0.05 and 0.5 mil in diameter: (1) less than 50 percent of all pores present are detected by the ferroxyl test; and (2) of this number, the larger the pore the more likely it will be detected.

Electrical discharge induced luminescence of solids at low temperatures, L. J. Schoen and R. E. Rebbert, J. Mol. Spect. 3, No. 4, 417 (1959).

Luminescence of condensed nitrogen-oxygen-argon systems at low temperatures has been excited by a-c and d-c discharge techniques. Typical atomic and molecular spectra obtained by these methods are described. The structure and wavelengths of the $\beta$ line group attributed to trapped oxygen atoms $\left({ }^{1} S \rightarrow 1 D\right)$ and the wavelengths of the $A$ and VegardKaplan band systems are compared in nitrogen and argon matrices. In argon a line group interpreted as the ${ }^{1} S \rightarrow^{3} P$ atomic oxygen transition has been observed. The principal mechanism of excitation under the conditions employed appears to be ion-electron bombardment of the deposited solid.

Program of the International Commission on radiological units and measurements, L. S. Taylor, L. H. Gray, and H. O. Wyckoff, 2d United Nations Intern. Conf. on the Peaceful Uses of Atomic Energy 21, Health and Safety: Dosimetry and Standards, $P / 2243 W H O$, p. 81 (1959).

A short review is given of the history of the ICRU, the program which resulted in its 1956 report, and the current program under way which will result in a new report next year. Special emphasis is placed upon the international character of the organization and its efforts to achieve international agreement on the measurement of radiation sources.

Spectrum of thin target bremsstrahlung bounded by a forward circular cone, J. H. Hubbell, J. Appl. Phys. 30, No. \%, 981 (1959).

The Schiff expression for the cross section per nucleus, for thin-target bremsstrahlung into the angular and energy ranges $d \theta_{0}$ and $d k$, is integrated analy tically over angle from zero to $\bar{\theta}_{0}$. Results are shown for the case $Z=78, E_{0}-m c^{2}=40$ Mev and $\bar{\chi}=0.5,1,2,4$, and 8 , where $E_{0}-m c^{2}$ is the kinetic energy of the incoming electron and $\bar{\chi}$ is the reduced angle $\vec{\theta}_{0} E_{0} / m c^{2}$. The fraction of the total cross section included in 
a cone of angle $\overline{\theta_{0}}$ can be approximated to within 20 percent by $\bar{\chi}^{2} /\left(1+\bar{\chi}^{2}\right)$. At $\bar{\chi}=1$, or $\vec{\theta}_{0}=0.723^{\circ}$, the ratio of energy delivered by photons above $\left(E_{0}-m c^{2}\right) / 2$ to that below is 9 percent greater than for the limiting spectral shape at $\bar{\theta}_{0}=0$, or that given by the Schiff expression at $\theta_{0}=0$, and 13 percent greater than for the spectrum integrated over all angles. For a target of finite thickness, multiple electron scattering should partially suppress the spectral dependence on -

Negative atomic ions, H. R. Johnson and F. Rohrlich, J. Chem. Phys. 30, 1068 (1959).

A semiempirical method is proposed for the computation of the electron affinities of negative atomic ions by extrapolation from the ionization potentials of the corresponding isoelectronic sequences. The method is based on a formula which can be justified on physical grounds and of which at least two parameters have direct physical interpretation. The results for the two short periods agree with experiments, with the important exception of $\mathrm{S}^{-}$. The method predicts a stable $\mathrm{N}^{-}$ion of small binding energy. The excited states and the fine-structure splitting can also be obtained by extrapolation based on theoretical formulas. The use of these formulas seems preferable to the method by Bates and Moisewitsch, but the results on excited states are the same.

The very low-frequency emissions generated in the earth's exosphere, R. M. Gallet, Proc. IRE, 47, No. 2, 211 (1959)

Naturally occurring, very low-frequency signals not associated with lightning discharges, and strongly correlated with solar activity, have been recognized nearly as long as the atmospheric whistlers which have their genesis in lightning discharges. Whereas whistlers have been satisfactorily explained, until recently these other phenomena have not. From the examination of a large quantity of high resolution spectograms, it has been deduced that a major fraction, if not all, of these other "noises" are excited in the exosphere by streams and bunches of high-speed ionized particles precipitating into the ionized atmosphere in the presence of the earth's magnetic field. The electromagnetic waves excited are then propagated in the manner of whistlers.

The excitation mechanism is similar to the operation of a traveling-wave tube. Two frequencies are simultaneously generated, and their values depend only on the three parameters: local electronic density in the exosphere, local magnetic field intensity, and particle velocity $\mathrm{V}$.

Reaction of hydrogen atoms with solid oxygen at $\mathbf{2 0}^{\circ} \mathbf{K}, \mathrm{R}$. Klein and M. D. Scheer, J. Chem. Phys. 31, No. 1, 278 (1959).

Hydrogen atoms, produced in the gas phase by thermal decomposition on a hot tungsten ribbon, react with solid oxygen at $20^{\circ} \mathrm{K}$. The primary reaction is most probably $\mathrm{H}+\mathrm{O}_{2} \rightarrow \mathrm{HO}_{2}$.

\section{Application of the Williams-Landel-Ferry equation to silicate glasses, A. B. Bestul, Glastech. Ber. (Frankfurt, Germany) 32 K, No. VI, 59 (1959).}

Viscosity data for silicate glasses in the glass transformation region have been fitted to the Williams-Landel-Ferry equation. The values resulting for one of the two constants are about the same as those obtained for organic glasses. The values for the second constant are much different from those for organic glasses. This second constant has been shown by Bueche to contain the volume coefficient of thermal expansion, $\alpha_{2}$, about the transformation temperature. If the applicable values of $\alpha_{2}$ are extracted from the second constant, the residues are about the same for silicate glasses and organic glasses. These findings confirm for silicate glasses the conclusion of Williams, Landel, and Ferry and of Bueche concerning the insensitivity to details of molecular structure of the properties of glass-forming substances which control their temperature dependence of molecular relaxation times.

Glow discharge spectra of copper and indium above aqueous solutions, $D$. E. Couch and $A$. Brenner, Tech. Notes, J. Electrochem. Soc. 106, 628 (1959).

An unusual glow discharge was produced between a tungsten electrode and an aqueous solution of cupric or indium salts. This glow was not produced by other salts or by a discharge to a fused salt bath containing copper.

Gaseous heat conduction at low pressures and temperatures, R. J. Corruccini, Vacuum $\mathbf{y - 8 ,} 19$ (Pergamon Press Ltd., London, England, 1959).

Over the large temperature differences that can exist in vacuum insulation of cryogenic devices some of the assumptions on which the formulas for free molecule heat conduction are based are not strictly valid. The consequences of this are discussed, and some incorrect usages are pointed out. Working formulas and auxiliary data on mean free paths and accommodation coefficients at eryogenic temperatures are presented.

Multiple ionization of sodium vapor by electron impact, V. H. Dibeler and R. M. Reese, J. Chem. Phys. 31, No. 1, 282 (1959).

Mass spectrometric studies are made of multiply-charged ions in sodium vapor. The results indicate that within a few volts of threshold, the probability of ionization increases as the $n$th power of the energy in excess of threshold, where $n$ is the number of charges on the ion. This is in agreement with Geltman's theoretical treatment of the threshold law for ionization by electrons.

Vibrational intensity distributions in the nitrogen afterglow, U. H. Kurzweg and $\mathrm{H}$. P. Broida, J. Mol. Spect. 3, No. 4, 388 (1959).

Nitrogen afterglows produced in a fast-flow system by an electrodeless discharge bave been studied as a function of pressure, flow, diluent, and temperature. Intensity measurements of the $\mathrm{N}_{2}$ 1st positive bands $\left(B^{3} \Pi-A^{3} \Sigma\right)$ responsible for the visible afterglow were obtained by means of a photoelectric recording monochromator. It is shown that the vibrational intensity distributions are not explainable by the generally accepted, preassociation model of the afterglow phenomena. High resolution scans depicting the relative intensity behavior of the $B^{3} \Pi-A^{3} \Sigma$ system under a variety of conditions are presented.

Influence of crystallographic orientation on the pitting of iron in distilled water, J. Kruger, $J$. Electrochem. Soc. 106, No. 8, 736 (1959).

The frequency of pitting on iron exposed to distilled water was found to depend on crystallographic orientation. The number of pits per unit area was highest for $\{110\}$.

A direct-reading viscometer, $\mathrm{M}$. R. Shafer, Instruments and Control Systems 32, No. \%, 1044 (1959).

Prototype instruments for determining directly the kinematic viscosity of liquids have been developed using the restrictionsin-series principle. Although not an absolute method, a simple calibration with liquids of known viscosities permits the subsequent measurement of the viscosities of other liquids by reading a single manometric column; no corrections or other calculations are required. Theory, construction, and operation of instruments having the range 0.5 to 5.0 centistokes are discussed. 
Journal of Research, Section 64D, No. 1, January-February 1960. go $_{0}$ cents.

Effect of antenna size on gain, bandwidth, and efficiency, R. G. Harrington.

Surface-wave resonance effect in a reactive cylindrical structure excited by an azial line source, A. L. Cullen. (See above abstract.)

Basic experimental studies of the magnetic field from electromagnetic sources immersed in a semi-infinite conducting medium, M. B. Kraichman. (See above abstract.)

A very-low-frequency antenna for investigating the ionosphere with horizontally polarized radio waves, R. S. Macmillan, W. V. T. Rusch, and R. M. Golden.

Effects of high-altitude nuclear explosions on radio noise, C. A. Samson.

Measured frequency spectra of vlf atmospherics. T. Obayashi.

Determination of the amplitude-probability distribution of atmospheric radio noise from statistical moments, W. G. Crichlow, C. J. Roubique, A. D. Spaulding, and W. M. Beery. (See above abstract.)

Measurements of coastal deviation of high-frequency radio waves, C. W. McLeish.

An exact earth-flattening procedure in propagation around a sphere. B. Y.-C. Koo and M. Katzin.

Limit of spatial resolution of refractometer cavities, W. J. Hartman. Conference on arctic communication.

Tropospheric scatter propagation and atmospheric circulations, W. F. Moler and D. B. Holden.

Layered earth propagation in the vicinity of Point Barrow, Alaska, G. M. Stanley.

Announcement of systematic ionospheric electron density data.

Tables of the bivariate normal distribution function and related functions. NBS AMS50, \$3.25.

Prediction of the cumulative distribution with time of ground wave and tropospheric wave transmission loss. Part 1. The prediction formula, P. L. Rice, A. G. Longley, and K. A. Norton, Tech. Note 15 (PB151374), \$1.50.

Analysis of ionospheric vertical soundings for electron density profile data. 1. Facilities for convenient manual reduction of ionograms, J. W. Wright and R. B. Norton, Tech. Note 14 (PB151373), 50 cents.

Analysis of ionospheric vertical soundings for electron density profile data. II. Extrapolation of observed electron density profiles above $h_{\max }$ F2, J. W. Wright, Tech. Note 19 (PB151378), 50 cents.

New approach in the theory of satellite orbits, J. P. Vinti, Phys. Rev. Letters 3, No. 1, 8 (1959).

Production of embossing plates from texture patterns by electroforming methods, J. P. Young and V. A. Lamb, Plating 46, 1033 (1959).

Method of evaluating the clinical effect of warping a denture: Report of a case, J. B. Woelfel and G. C. Paffenbarger, J. Am. Dental Assoc. 59, 250 (1959).

The sound transmission loss of some building construction, R. V. Waterhouse, R. D. Berendt, and R. K. Cook, Noise Control 5, No. 4, 40 (1959).

Cryogenic insulation, R. H. Kropschot, Am. Soc. Heating, Refrig. and Air-Conditioning Engrs. 1, No. 9, 48 (1959).

End plate modification of X-band TE011 cavity resonators, M. S. Thompson, F. E. Freethey, and D. M. Waters, IRE Trans. on Microwave Theory Tech. MTT-y, 388 (1959).
Evolution of amplified waves leading to transition in a boundary layer with zero pressure gradient, P. S. Klebanoff and K. D. Tidstrom, NASA Tech. Note D-195, 1 (1959).

Microwave reflectometer, G. F. Engen and R. W. Beatty, IRE Trans. on Microwave Theory Tech. MTT-\%, 351 (1959).

Penetration and diffusion of X-rays, U. Fano, L. V. Spencer, and M. J. Berger, Encyclopedia of Phys. 38, No. 2, 660 (1959).

Problems of the experimeter, W. J. Youden, Natl. Conv. Trans. Am. Soc. Quality Control, p. 41 (1959).

Some characteristics of VLF propagation using atmospheric waveforms, W. L. Taylor and L. J. Lange, Recent Advances in Atmospheric Electricity (Proc. 2d Conf. on Atmospheric Elec.), Portsmouth, New Hampshire, May 20 to 23, 1958, p. 609 (Pergamon Press, Inc., New York, N.Y., 1958).

The changing character of chemical research in government, E. Wichers, The Chemist XXXVI, No. 7, 260 (1959).

Study of the setting of plaster, K. D. Jorgensen and A. S. Posner, J. Dental Research, 38, No. 3, 491 (1959).

Optimum antenna height for ionospheric scatter propagation, R. G. Merrill, IRE Conv. Record \%, Pt. 1, 10 (1959).

Corrosion of type 310 stainless steel by synthetic fuel oil ash, H. L. Logan, Corrosion 15, 443t (1959).

Physical research, Pt. 2, G. C. Paffenbarger and W. Souder, J. Am. Dental Assoc. 58, 98 (1959).

The structure of electrolytic solutions, W. J. Hamer (John Wiley and Sons, Inc., New York, N.Y., 1959).

Width of cracks in concrete at the surface of reinforcing steel evaluated by means of tensile bond specimens, D. Watstein and R. G. Mathey, J. Am. Concrete Inst. 31, No. 1, 47 (1959).

Recent advances in cryogenic engineering, R. B. Jacobs, Am. Rocket Soc. ARS J. 29, 245 (1959).

Transistorized velocimeter for measuring speed in the sea, C. E. Tschiegg, J. Acoust. Soc. Am. 31, No. 7, 1038 (1959).

Inclusion theorems for congruence subgroups, $\mathbf{M}$. Newman and I. Reiner, Trans. Am. Math. Soc. 91, No. 3, 369 (1959).

Factors affecting modulation techniques for VHF scatter systems, J. W. Koch, IRE Trans. Commun. Systems CS-\%, No. 2, 77 (1959).

Calculated patterns of slotted elliptic-cylinder antennae, J. R. Wait and W. E. Mientka, Appl. Sci. Research B \%, 449 (1959).

Free radical chemistry, J. W. Moyer and A. M. Bass, Chem. and Eng. News p. 51 (Aug. 24, 1959).

Discussion of the papers of Messrs. Satterthwaite and Budne, W. J. Youden, Technometrics 1, No. 2, 157 (1959). 
A new aid for the rapid determination of absorption corrections by Albrecht's method, D. K. Smith, Acta Cryst. (Copenhagen, Denmark) 12, Pt. 6, 479 (1959).

Radiation attenuation data, H. O. Wyckoff, Radiation Hygiene Handbook, edited by H. Blatz, sec. 8, p. 1 (McGraw-Hill Book Co., New York, N.Y.) (1959).

Exposure standards and radiation protection regulations, L. S. Taylor, Radiation Hygiene Handbook, edited by H. Blatz, sec. 3, p. 2 (McGrawHill Book Co., New York, N.Y., 1959).

Properties of rutile (titanium dioxide), F. A. Grant, Rev. Modern Phys. 31, 646 (1959).

The interlaboratory evaluation of testing methods, J. Mandel and T. W. Lashof, ASTM Bull. No. 239, 53 (1959).

Measurements made by matching with known standards, W. J. Youden, W. S. Connor, and N. C. Severo, Technometrics 1, No. 3, 101 (1959).

Evacuated powder insulation for low temperatures, M. M. Fulk, Progress in Cryogenies-1, 65 (Heywood and Co., Ltd., London, England, 1959).
The following papers were published in the Proc. 5th Tech. Session on Bone Char, 1957 (Bone Char Research Project, Inc., Charlestown, Mass., 1959):

Introductory remarks, A. V. Astin, p. 1.

Analysis of liquid sugars, E. J. McDonald, p. 77.

Development of a new test for the abrasion hardness of bone char, F. G. Carpenter, p. 99.

Effects of controlled decarbonization on the performance of service synthad, F. W. Schwer, W. V. Loebenstein, and E. P. Barrett, p. 145 .

Some mechanisms of color and ash removal by bone char, A. Gee, p. 163

Survey of variations in use for conducting laboratory-scale column filteration tests, V. R. Deitz, p. 237.

Comparison of column decolorization experiments with theory, W. V. Loebenstein, p. 253.

Sugar retention by char, F. G. Carpenter, p. 279.

Removal or organic anions by bone char, V. R. Deitz and H. M. Rootare, p. 297.

*Publications for which a price is indicated (except for Technical Notes) are available only from the Superintendent of Documents, U.S. Government Printing Office, Washington 25, D.C. (foreign postage, one-fourth additional). Technical Notes are available only from the Office of Technical Services, U.S. Department of Commerce, Washington 25, D.C. (order by $P B$ number). Reprints from outside journals and the NBS Journal of Research may often be obtained directly from the author. 